\title{
Modeling research in recent years: special section on ECMFA 2017 and ECMFA 2018
}

\author{
Anthony Anjorin ${ }^{1} \cdot$ Alfonso Pierantonio $^{2} \cdot$ Salvador Trujillo ${ }^{1,2} \cdot$ Huascar Espinoza Ortiz ${ }^{1,2}$
}

Received: 30 July 2020 / Accepted: 30 July 2020 / Published online: 28 August 2020

(c) Springer-Verlag GmbH Germany, part of Springer Nature 2020

\section{Introduction}

The modeling community has made significant progress in recent years regarding developing software and systems with enhanced productivity and quality. Leveraging the critical entities emerging in the development process and their correspondences allows us to design, analyze, and develop software and systems relying on formalized notations that are amenable to computer-based automation. The European Conference on Modelling Foundations and Applications (ECMFA) is one of the primary scientific venues where almost any aspect of modeling is discussed among worldclass experts from academia and industry.

\section{Special section background and overview}

This special section aims to provide a representative sample of advanced research in modeling, model-based engineering, and model-driven engineering emerging from the 13th and 14th European Conference on Modelling Foundations and Applications (ECMFA) as part of STAF 2017 and 2018. The ECMFA follows a rigorous review process with each manuscript reviewed at least by three independent experts, followed by an in-depth discussion to finalize the technical program. Conflicts of interest were prevented based on author and abstract screening. All special section reviewers were recruited from the original pool of reviewers for the conference papers. Additional reviewers have also been added for the extended versions. Each special section paper has undergone multiple improvement cycles with associated rebuttal

\footnotetext{
Alfonso Pierantonio

alfonso.pierantonio@univaq.it

Anthony Anjorin

anthony.anjorin@iav.de

IAV GmbH, Carnotstraße 1, 10587 Berlin, Germany

2 DISIM - DEWS, Università degli Studi dell'Aquila, 67100

L'Aquila, Italy
}

letters. Finally, from the initial submissions we selected nine articles (three from the 2017 edition and six from 2018 one). The selected papers are briefly highlighted in the following:

- In their paper Integrated model-driven development of self-adaptive user interfaces, Enes Yigitbas, Ivan Jovanovikj, Kai Biermeier, Stefan Sauer, and Gregor Engels present a model-driven development approach for self-adaptive user interfaces that takes not only the development of the UI into account, but also context management and UI adaptation at runtime. These three coupled aspects are handled in an integrated manner using the standard UI modeling language IFML, combined with new modeling languages ContextML for context management, and AdaptML for UI runtime adaptation rules.

- In their contribution Automatic generation of UML profile graphical editors for Papyrus, Ran Wei, Athanasios Zolotas, Horacio Hoyos Rodriguez, Simos Gerasimou, Dimitris Kolovos, and Richard Freeman Paige show how metamodel annotations and model transformation techniques can help manage the complexity of Papyrus in the creation of UML profiles and their supporting editors. The approach is compared against manual UML profile specification and editor implementation demonstrating its effectiveness.

- In their paper A query-retyping approach to model transformation co-evolution, Adrain Rutle, Ludovico Iovino, Harald König, and Zinovy Diskin report an approach to metamodel/transformation co-evolution. Whenever metamodels undergo modifications, related model transformations may go out of sync. The approach is based on encoding the model transformation definition as a query-retyping combination and the evolution of the metamodels as applications of graph transformation rules.

- In his paper A verified catalog of OCL optimizations, Jesús Sánchez Cuadrado proposes a catalog of refactorings for OCL that aims to simplify OCL expressions that 
are automatically synthesized. The approach is evaluated for a large number of expressions and proved to be correct. Tool support is also provided.

- In their paper Incorporating measurement uncertainty into OCL/UML primitive datatypes, Manuel F. Bertoa, Loli Burgueño, Nathalie Moreno, and Antonio Vallecillo propose an extension of OCL/UML primitive datatypes that enables the representation of the uncertainty that comes from physical measurements or user estimates in models. An algebra of operations defined for the values of these types is proposed for dealing with uncertainty information.

- In their paper A semi-automated BPMN-based framework for detecting conflicts between security, dataminimization, andfairness requirements, Qusai Ramadan, Daniel Strüber, Mattia Salnitri, Jan Juerjens, Volker Riediger, and Steffen Staab present a BPMN-based security engineering framework. The approach aims to supports the design of business processes encompassing security, data minimization, and fairness requirements in terms of reusable conflict-free patterns. A case study featuring a healthcare management system is used to demonstrate the feasibility of the approach.

- In their paper Scalable modeling technologies in the wild: an experience report on wind turbines control applications development, Abel Gómez, Xabier Mendialdua, Konstantinos Barmpis, Gabor Bergmann, Jordi Cabot, Xabier de Carlos, Csaba Debreceni, Antonio Garmendia, Dimitris Kolovos, and Juan de Lara report lessons learned and their experience of applying some of the tools and results from the MONDO project to improve the processes of Ikerlan, a medium-sized technology center in Spain.
- In their contribution Model-driven process enactment for NFV systems with MAPLE, Sadaf Mustafiz, Omar Hassane, Guillaume Dupont, Ferhat Khendek, and Maria Toeroe report a process enactment approach to support automated process execution with Network Functions Virtualization systems as the target application domain. The approach is based on megamodels, and an integrated process modeling environment has been built into Papyrus for this purpose. The enactment environment transforms the process model to a model transformation chain and then orchestrates it with the use of megamodels.

- In their paper Modelling multi-criticality vehicular software systems: evolution of an industrial component model, Alessio Bucaioni, Saad Mubeen, Federico Ciccozzi, Antonio Cicchetti, and Mikael Sjödin report on systematically evolving the Rubus Component Model to support multi-core in modeling software with multicriticality for vehicular embedded systems. An important challenge addressed by the authors is that this evolution must be non-disruptive with respect to existing vehicular software assets.

Acknowledgements We are grateful to everyone who has made this special section possible. In particular, we are obliged to the referee for their invaluable contribution to thoroughly and thoughtfully review and re-review papers, to the authors for their hard work, and to the SoSyM Editorial Office for supporting this special issue. Last but not least, we would like to thank Martin Schindler for his competence, patience, and right-to-the-point attitude.

Publisher's Note Springer Nature remains neutral with regard to jurisdictional claims in published maps and institutional affiliations. 